\title{
An Interference Aware Routing Scheme Based on Channel Load for Multi-Radio Multi-Channel Wireless Mesh Networks
}

\author{
Jeong-Soo Kim, Sang-Hwa Chung, and Hyun-Suk Choi
}

\begin{abstract}
This paper focuses on the implementation and the verification of an interference aware routing scheme based on channel-load for multi-radio multi-channel wireless mesh network. Channel-load which is defined in IEEE 802.11k is channel consumption per time period of radio channel. Channel-load metric is able to recognize interference better than existing metrics in IEEE 802.11 networks. As a result, this routing metric achieves higher performance than other metrics in the environment with interference that has insufficient radio channel resources like wireless mesh network. Also, transmission of additional packets are not required to measure routing metric, and the status of wireless links is reflected accurately by using this metric, because it is calculated from channel busy time and measurement duration of profiling registers in the WALN interface. In this paper, we implemented the channel-load metric on WLAN mesh routers and verified the performance of the WLAN mesh network using this metric. The WLAN mesh network with the proposed routing scheme has improved both the throughput and the probability to select better route by about $30 \%$ than other schemes.
\end{abstract}

Index Terms-Channel-load, IEEE $802.11 s$, routing metric, routing scheme, wireless mesh network.

\section{INTRODUCTION}

Recently AP (Access Point)-based wireless networks are becoming more common due to rapid spread of mobile devices such as smart phones, smart pads, and notebooks. However, to extend AP-based wireless networks, a large number of APs and wired network infrastructures should be installed, because AP-based wireless networks have short coverage. Wireless mesh network is one of technology to solve the problem mentioned above. Wireless mesh network (WMN) has been drawing significant attention in recent years due to their flexibility in providing extensive wireless backbone. The main features of IEEE 802.11 WMN are processing routing in MAC (Medium Access Control) layer and forming network infrastructure with wireless multi-hop links. Because of those features, WLAN mesh network can provide routing efficiency in MAC layer and extendibility of multi-hop communication [1], [2].

Currently, as wireless networks are becoming more common, APs are installed in anywhere. Many mobile devices that use AP-based wireless networks share limited radio channels. For example, in South Korea, there are just

Manuscript received October 30, 2012; revised January 23, 2013.

The authors are with the Department of Computer Engineering, Pusan National University, Busan, Republic of Korea (e-mail: mankindy@pusan.ac.kr, shchung@pusan.ac.kr, choihs@pusan.ac.kr). three non-overlap radio channels in IEEE 802.11g and twelve non-overlap radio channels in IEEE 802.11a [3]. Thus, communication throughput of mobile devices is decreased due to interference between each other and sharing the limited radio channels. Especially, wireless mesh network suffers from both internal and external interferences, because wireless mesh network is a highly dense network of wireless mesh routers.

In this paper, we implemented the interference aware routing scheme based on channel-load that is channel usage per time unit of radio channel and verified the performance of multi-radio multi-channel wireless mesh networks using the interference aware routing scheme. The channel-load metric is calculated by a method defined in IEEE $802.11 \mathrm{k}$ [4].

The existing routing metrics such as hop-count, Expected Transmission count (ETX), Expected Transmission Time (ETT), Weighted Cumulative ETT (WCETT), and the air-time defined in IEEE 802.11s do not reflect interference environment, when the metrics were generated. Also, the existing routing metrics need to transmit additional packets to calculate the metrics. On the other hand, the proposed metric can recognize interference better than other metrics, because the proposed metric is generated from channel-load which reflects the status of wireless links with more accuracy. Besides, the proposed metric does not need to transmit additional packets, because it is calculated from channel busy time and measurement duration of profiling registers in the WLAN interface.

The remainder of this paper is organized as follows; Section II briefly introduces the existing routing metrics. Section III describes the routing scheme based on channel-load over WMN. Section IV presents the experimental environments and results. Finally, Section V concludes our work.

\section{RELATED WORK}

When a routing path is generated for data communication in WMN, existing WMN researches recommend the shortest path. However, hop-count metric which reflects only the length of the path is not suitable in WMN using multi-interfaces, because the bandwidth decrease due to the increase of hop-count is not significant in WMN using multi-interfaces unlike single interface.

ETX metric [5] that is calculated from packet loss rate reflects characteristics of wireless links. It means expected count of retransmission for data transmission. In ETX metric, all nodes should periodically broadcast probe packets. A neighbor node that received the prove packet sends the 
response to the sender. The sender calculates packet loss rate for the link between the sender and the receiver using response of probe packet. ETX metric is calculated as the following equation.

$$
\begin{aligned}
& p=\left(1-d_{f}\right) \times\left(1-d_{r}\right) \\
& \operatorname{ETX}=1 /(1-p)
\end{aligned}
$$

In Eq. (1), ' $d_{f}$ ' is the probability of successfully sending probe packets. ' $d_{r}$ ' means the probability of successfully receiving the response of probe packets. ETX is calculated from packet loss rate in Eq. (2). ETX metric is accumulated by increasing hops. Therefore, ETX metric reflects packet loss rate and route length.

ETT metric [6], [7] is improved from ETX metric. ETT metric reflects packet loss rate, route length, and link bandwidth between nodes. ETT metric is calculated as the following equation.

$$
E T T=E T X \times S / B
$$

In Eq. (3), ' $S$ ' is packet length and ' $B$ ' is link bandwidth. So, ETT metric can choose a path that has higher throughput and link bandwidth.

WCETT metric for multi-interfaces is calculated as the following equation.

$$
\begin{aligned}
& X_{i}=\sum_{\text {Hop } i \text { is on channel } j} E_{T T T_{i}} \quad 1 \leq j \leq k \\
& W C E T T=(1-\beta) \sum_{i=1}^{n} E T T+\beta \max _{1 \leq i \leq k} X_{i}
\end{aligned}
$$

In Eq. (4), ' $X_{i}$ ' is sum of ETT metrics for hops using the most common channel.

WCETT metric [8], [9] reflects packet loss rate and link bandwidth, because it is accumulated ETT metric. Also, WCETT metric reflects interference of the route, because it is accumulated ETT metric of the most common channel. WCETT metric can adjust the ' $\beta$ '. So, the metric is able to generate the suitable value for the environment. However, WCETT metric should know about channel allocation information of each link on the route. So, WCETT metric is just suitable for link-state routing protocols.

Air-time metric [10] defined in IEEE 802.11s calculates the metric using data rates among links and transmission error rates. Those existing metrics do not reflect enough interference and should need to transmit extra probe packets. However the proposed metric can reflect interference and does not need to transmit any additional packets.

\section{The Proposed Routing Scheme}

In this section, the routing scheme based on channel-load over WMN is explained.

\section{A. Routing Metric}

For establishing route in WMN, the routing scheme based on channel-load uses the channel-load metric. The metric is defined in IEEE $802.11 \mathrm{k}$ as the following equation. Its maximum value is 255 .

$$
\text { Channel Load }=\frac{\text { Channel Busy Time }}{\text { Measurement Duration }} \times 255
$$

The channel-load is channel consumption per time period of radio channel. The status of radio channel is reflected accurately by using this metric, because it is calculated from channel busy time and measurement duration of profiling registers in a WLAN interface. The channel busy time and the measurement duration mean channel consumption of radio channel of the WLAN interface and all other interfaces within interference range of the WLAN interface. Therefore, channel-load metric is able to recognize interference better than existing metrics. As a result, the path that has lower channel-load can obtain throughput better than others in interference environment.

IEEE 802.11 uses CSMA-CA (Carrier Sense Multiple Access with Collision Avoidance) and RTS (Request to Send) / CTS (Clear to Send) packets to communicate with others. In the communication method, the channel status that is busy or idle is monitored by the Clear Channel Assessment mechanism [3], which is based on the signal strength. Channel busy time and measurement duration that is calculated from the above channel status, and stored in profiling registers in the WLAN interface.

In this paper, we modified the mac80211 and the ath5k to implement the routing scheme based on channel-load in Compat-wireless [11] which is a Linux wireless driver package for WLAN interfaces. The mac80211 is a software MAC for WLAN communication and the ath5k is a Linux device driver for WiFi chipsets of Atheros Communications. The profiling registers in the $\mathrm{WiFi}$ chipset are used to calculate the channel-load metric when every second. In each network interface, AR5K_PROFCNT_CYCLE register records the total measurement time and AR5K_PROFCNT_RXCLR register records the channel busy time during the measurement time. The values of the profiling registers are periodically updated by the firmware of WiFi chipset. Therefore, we get the latest channel load metric for the WLAN interface.

\section{B. Routing Scheme}

The proposed routing scheme based on channel-load operates over HWMP (Hybrid Wireless Mesh Protocol) which is defined in the IEEE 802.11s standard. HWMP is based on distance vector routing which has just next-hop information for routing and consists of two modes: on-demand and pro-active mode.

The proposed routing scheme uses the on-demand of HWMP. The on-demand mode generates a route when any node tries to initiate communication with others, and the route information is updated periodically during the communication between nodes.

In this paper, we implemented the proposed routing scheme over on-demand mode of HWMP. The channel-load metric for the proposed routing scheme is called the Weighted Cumulative Channel Load (WCCL).

The first design goal of WCCL is to select a path which has the sparest bandwidth among the candidates. Therefore, $W C C L_{\text {part } 1}$ is defined as follows. For example, there are path 'A' which consists of one or more hops. Each hop of path 'A' shows different channel-loads respectively. The channel-load indicates the channel consumption per time unit of the radio 
channel. The throughput of the path is the most affected by the bottleneck link which has the largest channel-load value. Therefore, $W C C L_{\text {part } 1}$ uses the largest channel-load in the path as routing metric.

$$
W C C L_{\text {part } 1}=\max _{1 \leq i \leq n} X_{i}
$$

where ' $n$ ' is the number of hops on a path, ' $X_{i}$ ' is the channel-load of each hop.

The second design goal of WCCL is to select a path which has lower usage and lower hop-count among several candidates. The usage and hop-count is reflected by the total sum of channel-load in the path ' $A$ '. Therefore, $W C C L_{\text {part } 2}$ is expressed as follows.

$$
W C C L_{\text {part 2 }}=\sum_{i=1}^{n} X_{i}
$$

We can combine the two metrics described in Eq. (7) and (8) by taking their weighted average as shown below, where $\beta$ is a tunable parameter subject to $0 \leqslant \beta \leqslant 1$.

$$
W C C L=(1-\beta) \times \max _{1 \leq i \leq n} X_{i}+\beta \times \sum_{i=1}^{n} X_{i}
$$

There are two possible ways to explain (9). First, it can be explained as a tradeoff between global throughput and resource utilization on the path. The first term shows the channel usage in the specific hop which has the heaviest traffic of the path. The second term presents the sum of channel-load along all hops on the path. It reflects the total resource consumption and hop-count along the path. If $\beta$ is closer to the max, global throughput becomes more dominant factor. The weighted average can be considered to balance these two parts. Note that the above formula implicitly assumes that the network is not too heavily loaded. If all channel is being fully utilized, then simply considering overall resource consumption (setting $\beta=1$ ) may be preferable.

Second, (9) can be explained as a tradeoff between throughput and delay. The first term, since it represents the impact of bottleneck hops on this path, can be viewed as a measure of path throughput. The second term can be considered as a measure of the latency on this path. The weighted average can balance these two parts, too.

\section{EXPERIMENTAL SETTING AND RESUlTS}

In this paper, the proposed routing scheme is verified in real situation. Mesh routers [12], [13] for our experiments have $533 \mathrm{MHz}$ embedded processor and are ported with OpenWrt [14] which is the firmware program based on Linux kernel 2.6 for an embedded device such as network router or gateway. The proposed routing scheme is implemented by modifying the mac80211 of Compat-wireless.

Fig. 1 is the experimental environment to verify the proposed routing scheme. There are six mesh routers in the experimental environment.

As shown in Fig. 1, we consider only interference factor between L3 and L7 in the experimental environment. So, L1, L2, L4, L5, and L6 are allocated with non-overlap channels. On the other hand, L3 and L7 are set to use the same channel.
In this paper, we use Iperf [15], a general network-testing tool, to measure the performance. We did our experiments more than 10 times using UDP (User Datagram Protocol). In the experiments, we want to measure the performance of metrics according to various interference levels, when L3 and L7 have different transmission rates. For this experiment, we should be able to adjust transmission rate. Therefore, we use UDP instead of TCP (Transmission Control Protocol) in the experiments.

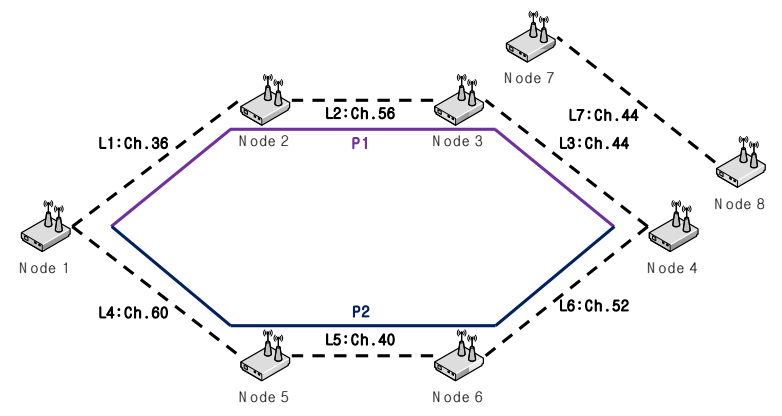

Fig. 1. Network topology for experiments.

In our experiments, L1, L2, L4, L5, and L6 have 10Mbps UDP traffics. L3 and L7 have different UDP traffic as defined in the following scenarios. All scenarios have 5Mbps UDP traffics from node 4 to node 1 .

1) Scenario 1 (S1) shown in Fig. 2; $\mathrm{S} 1$ does not have full channel-load at L3, when node 4 selects the P1 to reach node 1. In S1, L3 has background traffic smaller than L6. L3 and L7 have 5Mbps and 12Mbps UDP background traffic, respectively.

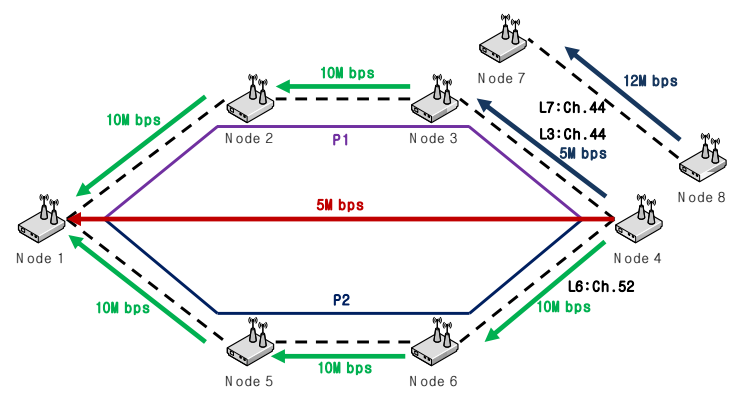

Fig. 2. Scenario 1

2) Scenario $2(\mathrm{~S} 2)$ shown in Fig. 3; S2 has full channel-load at L3, when node 4 selects the P1 to reach node 1 . In S2, L3 and L6 have same background traffic. L3 and L7 have 10Mbps UDP background traffic.

3) Scenario 3 (S3) shown in Fig. 4; S3 has full channel-load at L3, when node 4 selects the P1 to reach node 1 . In S3, L3 has background traffic smaller than L6. L3 and L7 have $5 \mathrm{Mbps}$ and $15 \mathrm{Mbps}$ UDP background traffic, respectively.

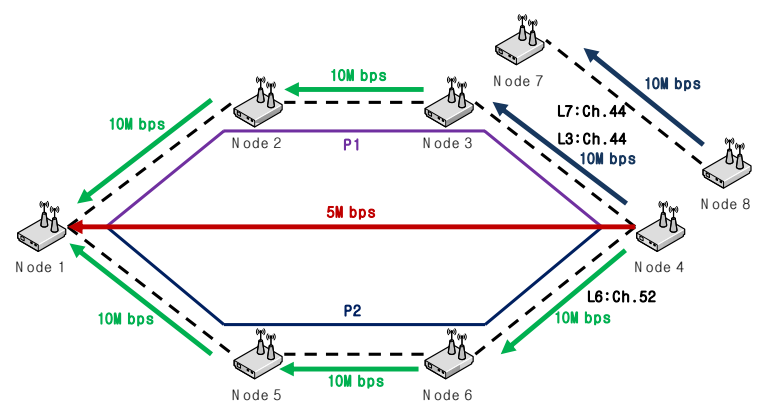

Fig. 3. Scenario 2 . 


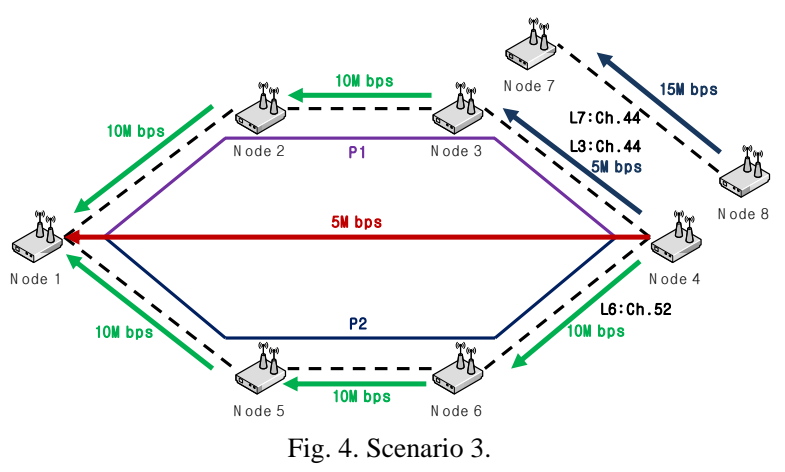

In the proposed metric, SUM_CL that reflects the total resource consumption will have the most impact on latency of the path. However, MAX_CL that reflects the channel-load of the link which has the most concentrated traffic may have the impact on throughput and latency of the path. Therefore, MAX_CL is more important than SUM_CL in the proposed metric. So, the $\beta$ is smaller than half. Thus, SUM_CL is the total sum of channel-load on the path and MAX_CL is the largest channel-load on the path.

Table I is the experimental result for four values of $\beta$ for $\mathrm{S} 2$. We did the experiments more than 10 times. As shown in Table I, when the $\beta$ is 0.15 , the average throughput is better than others. Therefore, we set the $\beta$ to 0.15 .

TABLE I: THROUGHPUT FOR FOUR VALUES OF BETA

\begin{tabular}{lllll}
\hline \hline$\beta$ & 0.05 & 0.15 & 0.25 & 0.35 \\
\hline Throughput (Mbps) & 4.22 & 4.35 & 4.32 & 4.15 \\
\hline \hline
\end{tabular}

$E T T$, air-time, and $W C C L$ are implemented and verified in our experiments. However, WCETT should know about the allocated channel information of each link on a route. Thus, WCETT is not suitable for distance vector routing protocols which utilize only next-hop information. So, we did not implement WCETT in our experiments.

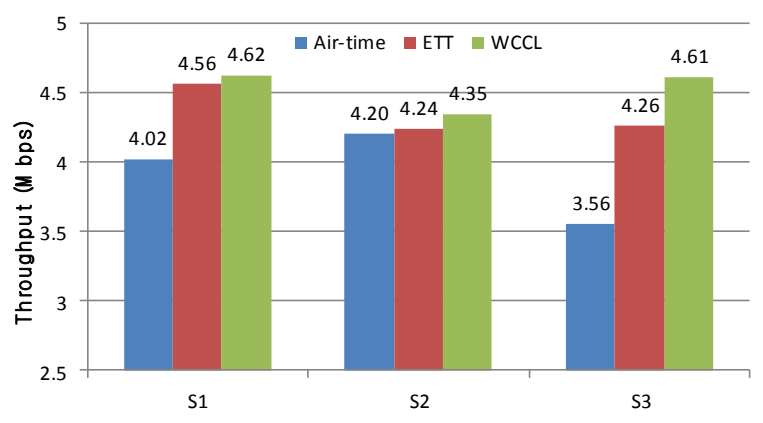

Fig. 5. Throughput for each metric.

Fig. 5 is the experimental result for each scenario. As shown in Fig. 5, WCCL has improved the performance by about $30 \%$ in maximum than other metrics. In WCCL, the probability to select $\mathrm{P} 1$ is higher compared to other metrics as shown in Fig. 7, because WCCL can recognize interference better than other metrics. In S1, Fig. 5 shows a large throughput gap between air-time metric and other metrics, because air-time metrics cannot recognize interference well compared with other metrics. In S3, Fig. 5 shows a large throughput gap between the WCCL metric and other metrics, because WCCL can recognize interference compared with other metrics. On the other hand, the air-time and ETT metric cannot recognize interference well, because these two metrics use packet error rate to generate the metrics. As shown in Fig. 6 , there are no correlation between interference and packet loss rate.

Fig. 6 shows packet loss rate of L3 vs. data transmission rate of L7 in experimental environment as shown in Fig. 1. As shown in Fig. 6, there is no correlation between data transmission rate of L7 and packet loss rate of L3, because L3 and $\mathrm{L} 7$ use CSMA/CA defined in IEEE 802.11. Thus, the two links avoid collision with each other. Therefore, packet loss rate of L3 is not affected by interference.

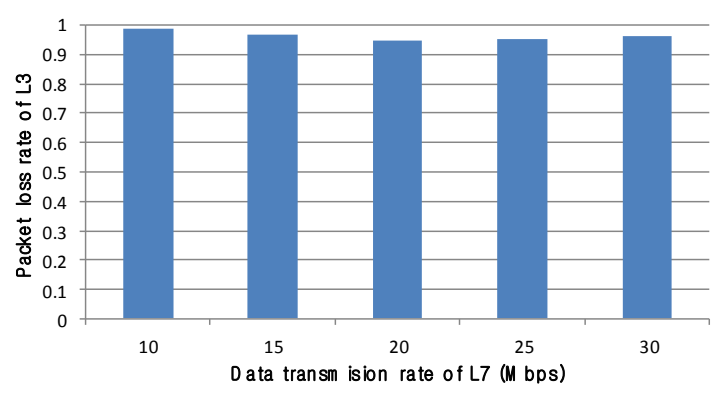

Fig. 6. Packet loss rate of L3 vs. data transmission rate of L7.

Fig. 7 shows the probability to select better route for each scenario in the same situation with Fig. 5. As shown in Fig. 7, WCCL has higher probability by about $30 \%$ than other metrics. Because, WCCL can recognize interference better than other metrics, So, WCCL can select good path and has the performance better than other metrics.

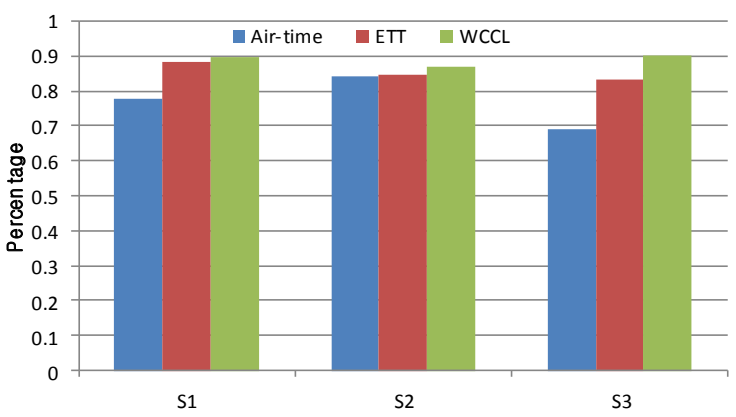

Fig. 7. Probability to select better route for each metric.

\section{CONCLUSION}

This paper focuses on the implementation and the verification of an interference aware routing scheme based on channel-load for multi-radio multi-channel wireless mesh network. The channel-load metric is able to recognize interference better than existing metrics in IEEE 802.11 networks. As a result, this routing metric achieves higher performance than other metrics in the environment with interference that has insufficient radio channel resources like wireless mesh network. Also, transmission of additional packets are not required to measure routing metric, and the status of wireless links is reflected accurately by using this metric, because it is calculated from channel busy time and measurement duration of profiling registers in the WALN interface. In this paper, we implemented the channel-load metric on WLAN mesh routers and verified the performance of the WLAN mesh network using this metric. The WLAN 
mesh network with the proposed routing scheme has improved both the throughput and the probability to select better route by about $30 \%$ than other schemes.

\section{REFERENCES}

[1] S. M. Faccin, C. Wijting, J. Kenckt, and A. Damle, "Mesh WLAN networks: concept and system design," IEEE Wireless Communications, vol. 13, no. 2, pp. 10-17, April 2006.

[2] I. F. Akyildiz, X. Wang, and W. Wang, "Wireless mesh networks: a survey," Computer Networks and ISDN Systems, vol. 47, no. 4, pp. 445-487, March 2005.

[3] IEEE Standard for Information Technology-Telecommunications and information exchange between systems-Local and metropolitan area networks-Specific requirements, Part 11: Wireless LAN Medium Access Control (MAC) and Physical Layer (PHY) Specifications, 802.11-2007.

[4] IEEE Standard for Information Technology- Telecommunications and information exchange between systems- Local and metropolitan area networks- Specific requirements, Part 11: Wireless LAN Medium Access Control (MAC) and Physical Layer (PHY) Specifications Amendment 1: Radio Resource Measurement of Wireless LANs, $802.11 \mathrm{k}-2008$

[5] D. S. J. D. Couto, D. Aguayo, J. C. Bicket, and R. Morris, "A high-throughput path metric for multi-hop wireless routing," in Proc. 2003 MobiCom Conf., September 2003, pp.134-146.

[6] P. M. Esposito, M. Campista, I. M. Moraes, L. Costa, O. Duarte, and M. G. Rubinstein, "Implementing the expected transmission time metric for olsr wireless mesh networks," in Proc. Wireless Days, WD '08, 1 st IFIP, November 2008, pp. 1-5,

[7] S. Biaz, B. Qi, and Y. M. Ji, "Improving expected transmission time metric in multi-rate multi-hop networks," in Proc. 2008 CCNC, January 2008, pp. 533-737.

[8] R. Draves, J. Padhye, and B. Zill, "Routing in multi-radio, multi-hop wireless mesh networks," in Proc. 2004 MobiComConf., September 2004, pp. $114-128$

[9] L. Ma and M. K. Denko, "A routing metric for load-balancing in wireless mesh networks," in Proc. AINA 2007, May 2007, pp. 409-414.

[10] IEEE Standard for Information Technology--Telecommunications and information exchange between systems--Local and metropolitan area networks--Specific requirements, Part 11: Wireless LAN Medium Access Control (MAC) and Physical Layer (PHY) specifications Amendment 10: Mesh Networking, 802.11s-2011.
[11] Compat-wireless. [Online]. Available: http://www.linuxwireless.org/

[12] J. S. Kim, S. H. Chung, Y. S. Lee, C. W. Ahn, W. S. Kim, and M. S Jung, "Design and implementation of a wlan mesh router based on multipath routing," in Proc. 2011 ICOIN Conf., January 2011, pp. $154-159$

[13] J. S. Kim, S. H. Chung, C. W. Ahn, and W. S. Kim, "Implementing channel-load aware routing scheme for IEEE 802.11 mesh networks," in Proc. 2011 WiMobConf., October 2011, pp. 525-528.

[14] OpenWrt. [Online]. Available: https://www.openwrt.org/

[15] Iperf. [Online]. Available: http://www.sourceforge.net/projects/iperf/

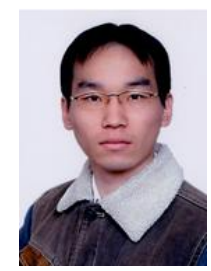

Jeong-SooKim received the B.S., and M.S. degrees in computer engineering from Pusan National University, Busan, Korea, in 2006 and 2008, respectively. He is currently a Ph.D. course in the Department of Computer Engineering, Pusan National University. Mr. Kim's research interests include wireless communications, wireless mesh network, embedded system, and embeddedoperating system.

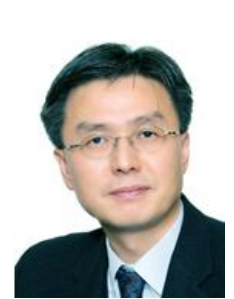

Sang-Hwa Chung received the B.S. degree in electrical engineering from Seoul National University in 1985, the M.S. degree in computer engineering from Iowa State University in 1988, and the Ph.D. degree in computer engineering from the University of Southern California in 1993. Since 1994, he has been with Pusan National University, where he is currently a Professor in the Department of Computer Engineering, Pusan National University. Prof. Chung's research interests include wired/wireless network, RFID system, embedded system, computer architecture, and high-performance computing.

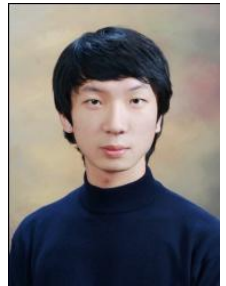

Hyun-Suk Choi received the B.S.degrees in computer engineering from Pusan National University, Pusan, Korea, in 2012, respectively. $\mathrm{He}$ is currently a Mastercourse in the Department of Computer Engineering, Pusan National University. Mr. Choi's research interests include wireless communications, wireless mesh network, Software defined network, embedded system, and embeddedoperating system. 